University of Nebraska - Lincoln

DigitalCommons@University of Nebraska - Lincoln

Faculty Publications from the Center for Plant

Science Innovation

Plant Science Innovation, Center for

$11-9-2007$

\title{
Comment on "A G Protein-Coupled Receptor Is a Plasma Membrane Receptor for the Plant Hormone Abscisic Acid"
}

Christopher Johnston

University of North Carolina, Chapel Hill

Brenda Temple

University of North Carolina, Chapel Hill

Jin-Gui Chen

University of British Columbia, Vancouver, BC V6T 1Z4, Canada

Yajun Gao

University of British Columbia, Vancouver, BC V6T 1Z4, Canada

Etsuko N. Moriyama

University of Nebraska - Lincoln, emoriyama2@unl.edu

See next page for additional authors

Follow this and additional works at: https://digitalcommons.unl.edu/plantscifacpub

Part of the Plant Sciences Commons

Johnston, Christopher; Temple, Brenda; Chen, Jin-Gui; Gao, Yajun; Moriyama, Etsuko N.; Jones, Alan M.; Siderovski, David; and Willard, Francis, "Comment on "A G Protein-Coupled Receptor Is a Plasma Membrane Receptor for the Plant Hormone Abscisic Acid"'" (2007). Faculty Publications from the Center for Plant Science Innovation. 30.

https://digitalcommons.unl.edu/plantscifacpub/30

This Article is brought to you for free and open access by the Plant Science Innovation, Center for at DigitalCommons@University of Nebraska - Lincoln. It has been accepted for inclusion in Faculty Publications from the Center for Plant Science Innovation by an authorized administrator of DigitalCommons@University of Nebraska Lincoln. 


\section{Authors}

Christopher Johnston, Brenda Temple, Jin-Gui Chen, Yajun Gao, Etsuko N. Moriyama, Alan M. Jones, David Siderovski, and Francis Willard 


\section{Comment on "A G Protein-Coupled Receptor Is a Plasma Membrane Receptor for the Plant Hormone Abscisic Acid"}

Christopher A. Johnston, ${ }^{1}$ Brenda R. Temple, ${ }^{2}$ Jin-Gui Chen, ${ }^{4}$ Yajun Gao, ${ }^{4}$ Etsuko N. Moriyama, ${ }^{5}$ Alan M. Jones, ${ }^{1,3}$ David P. Siderovski, ${ }^{1}$ Francis S. Willard ${ }^{1}$

${ }^{1}$ Department of Pharmacology, University of North Carolina, Chapel Hill, NC 27599, USA; ${ }^{2}$ Structural Bioinformatics Core Facility, University of North Carolina, Chapel Hill, NC 27599, USA; ${ }^{3}$ Department of Biology, University of North Carolina, Chapel Hill, NC 27599, USA; ${ }^{4}$ Department of Botany, University of British Columbia, Vancouver, BC V6T 1Z4, Canada; ${ }^{5}$ School of Biological Sciences and Plant Science Initiative, University of Nebraska-Lincoln, Lincoln, NE 68588-0660, USA

Corresponding authors — Alan M. Jones, email: alan_jones@unc.edu ; David P. Siderovski, email dsiderov@med.unc.edu ; Francis S. Willard, email fwillard@med.unc.edu

Abstract: Liu et al. (Reports, March 23, 2007, p. 1712) reported that the Arabidopsis thaliana gene GCR2 encodes a seven-transmembrane, G protein-coupled receptor for abscisic acid. We argue that GCR2 is not likely to be a transmembrane protein nor a G protein-coupled receptor. Instead, GCR2 is most likely a plant homolog of bacterial lanthionine synthetases.

G protein-coupled receptors (GPCRs) are commonly used by eukaryotic organisms for signal processing and homeostasis, but recognition of a bona fide plant GPCR has been elusive. Liu et al. (1) recently reported that the Arabidopsis thaliana gene GCR2 (TAIR gene name At1g52920) encodes a 401-amino acid GPCR for abscisic acid. Liu et al. predicted GCR2 as a seventransmembrane protein (7TM), using the TMpred and DAS programs, but did not report score thresholds to evaluate the confidence of these predictions. TMpred and DAS are known to erroneously predict transmembrane helices within soluble proteins $(55 \%$ and $83 \%$ false positive rates, respectively) (2). A newer version of DAS (the "DAS-TMfilter server"), containing a filter for false-positive predictions (http://mendel.imp.ac.at/sat/ DAS/DAS.html), does not predict transmembrane regions within GCR2. Two other algorithms, TMHMM2.0 (http:// www.cbs.dtu.dk/services/TMHMM) and SOSUI (http:/ / bp.nuap.nagoya-u.ac. ip/sosui), also do not predict transmembrane helices in GCR2. Both TMHMM2.0 and SOUSI are robust transmembrane helix predictors with low false-positive rates (1\% and $3 \%$, respectively) (2). A diverse set of protein classification methods was recently used to identify potential Arabidopsis 7TM proteins, but GCR2 was not among them (3).

BLAST (4) analysis of GCR2 indicates significant sequence similarity to bacterial [expect $(\mathrm{E})$ value, $2 \times 10^{-7}$ ], plant $\left(8 \times 10^{-}\right.$ $\left.{ }^{153}\right)$, human $\left(2 \times 10^{-68}\right)$, murine $\left(3 \times 10^{-69}\right)$, and insect $\left(3 \times 10^{-53}\right)$ lanthionine synthetase (LanC) proteins. Prokaryotic LanC enzymes produce cyclized antimicrobial peptides (5). The function of the eukaryotic LanC proteins is unknown. Significant sequence similarities between GCR2 and various prokaryotic and eukaryotic LanC proteins (Figure 1) indicate that these proteins belong to an evolutionarily conserved protein family. Predicting the tertiary structure of GCR2, using the protein-fold recognition algorithim PHYRE (http://www.sbg.bio.ic.ac.uk/ phyre),

A

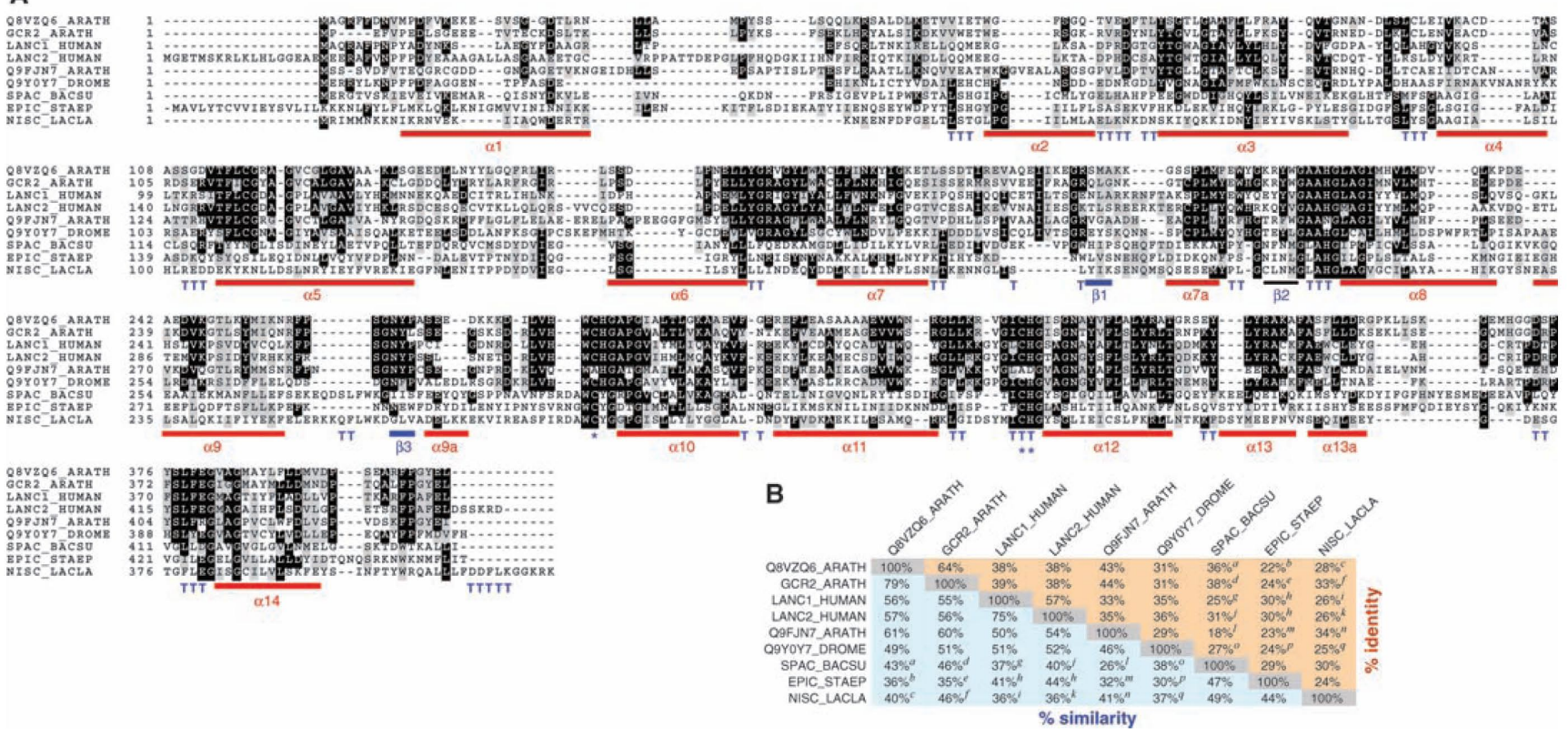

Figure 1. GCR2 is a member of the LanC protein superfamily. (A) Multiple sequence alignment of GCR2 and LanC family proteins. Secondary structures observed in the NisC crystal structure are denoted with the 14 major alpha helical regions ( $\alpha 1$ to $\alpha 14)$ of NisC underlined in red, the $\beta$ strands ( $\beta 1-3$ ) of the SH2-like "extended" domain underlined in blue, and residues involved in interhelix turns denoted by blue Ts. Conserved zinc-coordinating residues are denoted by asterisks. Proteins are denoted by their Swiss-Prot identifiers, except for GCR2 (GenBank accession NP_175700). Information for this figure was obtained from the PDB file 2G0D and Li et al. (6). Species abbreviations are ARATH (A. thaliana), BACSU (Bacillus subtilis), DROME (Drosophila melanogaster), HUMAN (Homo sapiens), LACLA (L. lactis), and STAEP (Staphylococcus epidermidis). (B) Percentage identity (orange boxes) and percentage similarity (blue boxes) from pairwise BLAST comparisons of indicated protein sequences using the BLOSUM45 matrix (4), except where the footnotes indicate identity and similarity statistics alternatively obtained from the BESTFIT algorithm (Accelrys GCG package) over the following subspans (aa = amino acids) of the indicated protein sequences: ${ }^{a}, 71$ aa; ${ }^{b}, 123$ aa; ${ }^{c}, 183$ aa; ${ }^{d}, 66$ aa; ${ }^{e}, 93$ aa; ${ }^{f}, 117$ aa; ${ }^{g}, 366$ aa; ${ }^{h}, 74$ aa; ${ }^{i}, 113$ aа; ${ }^{j}, 94$ aa; $^{k}, 355$ aa; ${ }^{l}, 72$ aa; $^{m}, 110$ aa; $^{n}, 65$ aa; $^{\circ}, 292$ aa; ${ }^{p}, 55$ aa; ${ }^{q}, 260$ aa. 


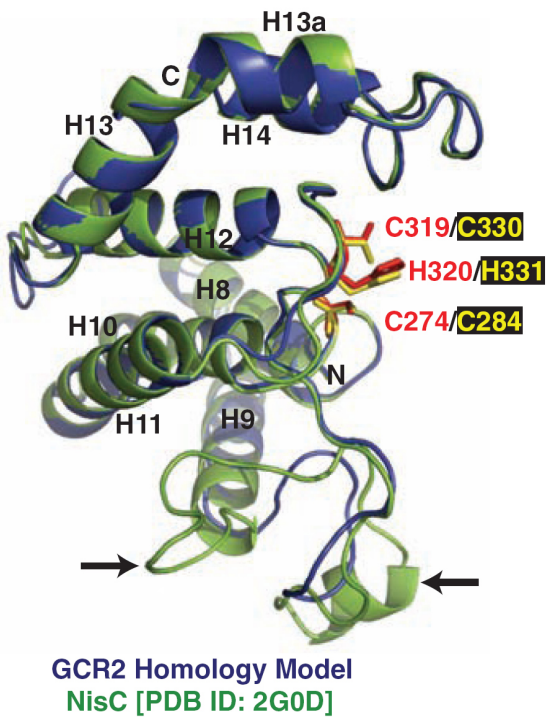

Figure 2. GCR2 has a predicted tertiary structure consistent with a LanC protein. BLASTP search against the structural database (http://www. rcsb.org), using GCR2 as the query, identified nisin cyclase (NisC, PDB ID: 2G0D) as the only structural homolog producing a statistically significant alignment [expect (E) value, $4 \times$ $10^{-4}$. Significant sequence similarity was noted between amino acids 216 and 282 of GCR2 and amino acids 209 and 386 of NisC. A homology model of GCR2 (amino acids 216 to 282) was then generated using Insight-II (http://www accelrys.com/products/insight). Shown is a superposition of the GCR2 homology model (blue) and the corresponding region of $\mathrm{NisC}$ (green). The $\mathrm{N}$ and $\mathrm{C}$ termini are labeled accordingly. Alpha helices observed in the $\mathrm{NisC}$ structure are denoted H8 to H14. Arrows indicate two segments in which NisC contains extended inserts relative to GCR2 and are the only areas of the superposition that diverge between the molecules. The proposed catalytic residues are indicated in NisC (yellow sticks) and GCR2 (red sticks). The superposition and image were generated using PyMol (DeLano Scientific, Palo Alto, CA, USA).

indicates that GCR2 is most likely an $\alpha$ $\alpha$ toroid protein. This fold is a defining structural characteristic of LanC proteins, terpenoid cyclases, glycosidases, and farnesyl transferases $(5,6)$.

We created a homology model of GCR2 based on the crystal structure of the Lactococcus lactis LanC protein, nisin cyclase (NisC) (Figure 2) (6). This homology model has a Profiles-3D self-compatibility score of $27.6 \%$, indicating a valid model with robust statistical confidence (7). The core $\alpha$-helices of NisC superimpose very well to those of the GCR2 homology model (Figure 2), with an overall root mean square deviation of $4.0 \AA$. The zinc-coordinating residues of $\mathrm{NisC}$, important for cysteine cyclization, are conserved in the primary sequence (Figure $1 \mathrm{~A})$, and these residues in NisC superimpose well with corresponding residues
A

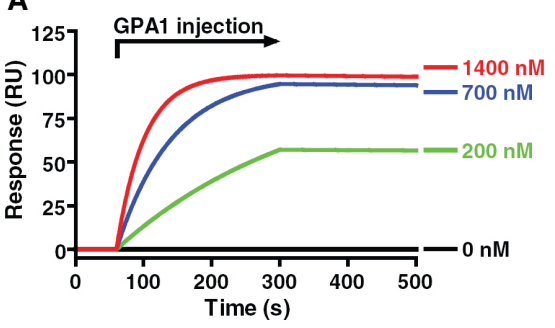

B

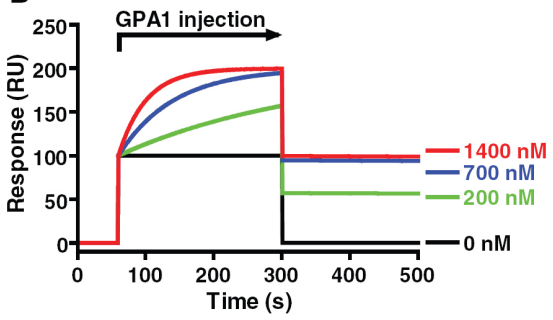

Figure 3. Simulated surface plasmon resonance binding curves for a $2 \mathrm{nM}$ affinity interaction between GPA1 and GCR2. Simulations of GPA1 binding to immobilized GCR2, using the rate constants published by Liu et al. $\left(\mathrm{k}_{\mathrm{a}}=1.77 \times 10^{4}\right.$ $\left.\mathrm{M}^{-1} \mathrm{~s}^{-1} ; \mathrm{k}_{\mathrm{d}}=3.9 \times 10^{-5} \mathrm{~s}^{-1}\right)$. Simulated injections are plotted for four different concentrations of GPA1 as reported by Liu et al. (1). Arrow indicates the injection time course and corresponding association phase. Simulated sensorgrams were generated using BIAeval 3.2 software (GE Healthcare, Uppsala, Sweden), using the 1:1 Langmuir model with maximum binding of 100 RU. (A) Simulated sensorgrams for an interaction that has no bulk buffer shift. (B) Simulated sensorgrams for an interaction occurring with a bulk buffer shift of 100 RU.

of the GCR2 model (Figure 2). This analysis provides a structural argument that GCR2 is a member of the LanC protein superfamily, not the GPCR superfamily.

Notably, a mammalian LanC homolog (LANCL1) was originally misidentified as a GPCR (GPR69A/p40) (8). In subsequent studies, the authors determined that GPR69A was in fact a LanC ortholog and renamed this protein LANCL1 (9). Biochemical studies confirmed LANCL1 to be a peripheral membrane protein (9). Subsequently, the related protein LANCL2 was suggested to be membrane localized due to both myristoylation and lipid binding (10). These data from orthologous proteins suggest that GCR2 is likely to be a peripheral membrane protein. Further evidence against GCR2 having a 7TM topology is provided by the split ubiquitin assays of Liu et al. showing that GPA1-Cub interacts equally well with both $\mathrm{N}$ - and C-terminal fusions of GCR2 to NubG (1). These results are incompatible with the GCR2 $\mathrm{N}$ terminus being extracellular, as is the case with all known GPCRs, and are incompatible with GCR2 having an odd number of transmembrane spans.
Liu et al. reported solubilizing recombinant GCR2 from Escherichia coli using $0.1 \%$ Triton-X100 and purifiying GCR2 to homogeneity. The apparent ease of this purification and the methods used are generally contrary to the known arduous biochemistry of GPCR purification, given 7TM helices (11), but are entirely consistent with purifying a soluble cytosolic protein from $E$. coli. In vitro proteinprotein interaction was reported between GCR2 and the Arabidopsis Ga subunit GPA1 using surface plasmon resonance (SPR) (1). However, the presented SPR data are not representative of a bona fide interaction (12). Indeed, the data clearly demonstrate an absence of any GCR2/ GPA1 interaction, as GPA1 binding to GCR2 is equivalent to that of the negative control BSA [figure S3 in (1)]. The presented sensorgrams are most likely bulk shift artifacts normally corrected by negative control subtraction (12). We were unable to determine how Liu et al. (1) measured their rate constants. However, simulated SPR sensorgrams based on their reported values (Figure 3) clearly demonstrate a discrepancy between the data of Liu et al. (1) and expected SPR results (12) based on their reported rate constants. The reported off-rate constant $\left(3.9 \times 10^{-5} \mathrm{~s}^{-1}\right)$ suggests that the GCR2/ GPA1 complex has a binding half-life of 5 hours, thoroughly inconsistent with the raw data presented by Liu et al. (1), and also suggesting that a surface regeneration step would be necessary to obtain reliable dose-response data.

The classical in vitro assay for GPCR/ $\mathrm{G} \alpha$ coupling is demonstration of agonistpromoted guanine nucleotide exchange factor activity, either by GTP $\gamma S$ binding or steady-state GTPase activity (13). Reconstituting interactions between $\mathrm{G} \alpha$ subunits and their cognate GPCRs typically requires lipid-modified $\mathrm{G} \alpha$ and $\mathrm{G} \beta \gamma$ subunits and a model membrane (13). The binding of G $\alpha$ and G $\beta \gamma$ to GPCRs is synergistic, whereas isolated subunits have low affinity for receptor (14). These considerations were not addressed by Liu et al. (1). In summary, while it is possible that GCR2 is both an intracellular receptor for abscisic acid and a G protein modulator, we conclude that GCR2 is neither a transmembrane protein nor a $G$ protein coupled receptor, but rather is an Arabidopsis homolog of bacterial lanthionine synthetases.We recommend that any putative plant GPCR be rigorously characterized as a bona fide G protein coupled receptor using in vitro biochemical methods for demonstrating $G$ protein coupling and activation that have been well-established for the analysis of mammalian GPCRs. 


\section{References and Notes}

1. X. Liu et al., Science 315, 1712 (2007).

2. S. Moller, M. D. Croning, R. Apweiler, Bioinformatics 17, 646 (2001).

3. E. N. Moriyama, P. K. Strope, S. O. Opiyo, Z. Chen, A. M. Jones, Genome Biol. 7, R96 (2006).

4. S. F. Altschul, W. Gish, W. Miller, E. W. Myers, D. J. Lipman, J. Mol. Biol. 215, 403 (1990).

5. D. W. Christianson, Science 311, 1382 (2006).

6. B. Li et al., Science 311, 1464 (2006).
7. R. Luthy, J. U. Bowie, D. Eisenberg, Nature 356, 83 (1992).

8. H. Mayer, J. Breuss, S. Zeigler, R. Prohaska, Biochim. Biophys. Acta 1399, 51 (1999).

9. H. Bauer, H. Mayer, A. Marchler-Bauer, U. Salzer, R. Prohaska, Biochem. Biophys. Res. Commun. 275, 69 (2000).

10. C. Landlinger, U. Salzer, R. Prohaska, Biochim. Biophys. Acta 1758, 1759 (2006).

11. V. Sarramegn, I. Muller, A. Milon, F.
Talmont, Cell. Mol. Life Sci. 63, 1149 (2006).

12. R. L. Rich, D. G. Myszka, J. Mol. Recognit. 19, 478 (2006).

13. E. M. Parker, K. Kameyama, T. Higashijima, E. M. Ross, J. Biol. Chem. 266, 519 (1991).

14. W. A. Clark, X. Jian, L. Chen, J. K. Northup, Biochem. J. 358, 389 (2001).

15. We thank S. K. Nair (University of Illinois at Urbana-Champaign) for valuable discussions. 